\title{
Necrostatin-1 Ameliorates Neutrophilic Inflammation in Asthma by Suppressing MLKL Phosphorylation to Inhibiting NETs Release
}

\section{OPEN ACCESS}

Edited by:

Fabrice Cognasse,

Groupe Sur L'immunité Des Muqueuses Et Agents Pathogènes (GIMAP), France Samithamby Jey Jeyaseelan,

Louisiana State University,

United States

Mausita Karmakar

Case Western Reserve University,

United States

${ }^{*}$ Correspondence:

K. F. Lai

Klai@163.com

E. W. Sun

sunew@smu.edu.cn

${ }^{\dagger}$ These authors have contributed equally to this work

Specialty section: This article was submitted to Inflammation,

a section of the journal

Frontiers in Immunology

Received: 30 December 2019 Accepted: 24 March 2020

Published: 24 April 2020

Citation:

Han X, Jie H, Wang J, Zhang $X$, Wang J, Yu C, Zhang J, He J, Chen J, Lai K and Sun E (2020) Necrostatin-1 Ameliorates Neutrophilic Inflammation in Asthma by Suppressing MLKL

Phosphorylation to Inhibiting NETS Release. Front. Immunol. 11:666 doi: 10.3389/fimmu.2020.00666

\begin{abstract}
Xinai Han ${ }^{1,2+}$, Hongyu Jie ${ }^{1,2 t}$, Jinhong Wang ${ }^{3}$, Xiaoming Zhang ${ }^{3}$, Jun Wang ${ }^{4}$, Chunxiao Yu ${ }^{1}$, Jialin Zhang ${ }^{1}$, Juan He ${ }^{5}$, Junqi Chen ${ }^{6}$, Kefang Lai ${ }^{7 *}$ and Erwei Sun ${ }^{1,2 *}$

${ }^{1}$ Department of Rheumatology and Immunology, The Third Affiliated Hospital, Southern Medical University, Guangzhou, China, ${ }^{2}$ Guangdong Provincial Key Laboratory of Bone and Joint Degeneration Diseases, The Third Affiliated Hospital, Southern Medical University, Guangzhou, China, ${ }^{3}$ Department of Respiratory and Critical Care Medicine, The Third Affiliated Hospital, Southern Medical University, Guangzhou, China, ${ }^{4}$ Department of Respiration, Nan Fang Hospital, Southern Medical University, Guangzhou, China, ${ }^{5}$ Department of Rheumatology and Immunology, Peking University Shenzhen Hospital, Shenzhen, China, ${ }^{6}$ Department of Rehabilitation Medicine, The Third Affiliated Hospital, Southern Medical University, Guangzhou, China, ${ }^{7}$ State Key Laboratory of Respiratory Disease, Guangzhou Institute of Respiratory Disease, The First Affiliated Hospital, Guangzhou Medical College, Guangzhou, China
\end{abstract}

Neutrophilic inflammation occurs during asthma exacerbation, and especially, in patients with steroid-refractory asthma, but the underlying mechanisms are poorly understood. Recently, a significant accumulation of neutrophil extracellular traps (NETs) in the airways of neutrophilic asthma has been documented, suggesting that NETs may play an important role in the pathogenesis. In this study, we firstly demonstrated that NETs could induce human airway epithelial cell damage in vitro. In a mouse asthmatic model of neutrophil-dominated airway inflammation, we found that NETs were markedly increased in bronchoalveolar lavage (BAL), and the formation of NETs exacerbated the airway inflammation. Additionally, a small-molecule drug necrostatin-1 (Nec-1) shown to inhibit NETs formation was found to alleviate the neutrophil-dominated airway inflammation. Nec-1 reduced total protein concentration, myeloperoxidase activity, and the levels of inflammatory cytokines in BAL. Finally, further experiments proved that the inhibition of Nec-1 on NETs formation might be related to its ability to inhibiting mixed lineage kinase domain-like (MLKL) phosphorylation and perforation. Together, these results document that NETs are closely associated with the pathogenesis of neutrophilic asthma and inhibition of the formation of NETs by Nec- 1 may be a new therapeutic strategy to ameliorate neutrophil-dominated airway inflammation.

Keywords: neutrophilic inflammation, neutrophil extracellular traps, Necrostatin-1, MLKL, asthma

\section{INTRODUCTION}

Asthmatic inflammation is dominated by the accumulation of eosinophils, neutrophils, or both, in the airways. Approximately half of the patients with asthma have eosinophilic inflammation, while the rest are characterized by increased number of neutrophils in sputum (1). Eosinophilic inflammation is commonly considered as type 2 inflammation and sensitive to 
inhaled corticosteroids, while Neutrophilic inflammation in asthma occurs in the absence of Th2 cytokines IL-4, IL5, and IL-13, and insensitive to inhaled corticosteroids (2). In patients with acute or persistent asthma, the number of neutrophils is increased and correlates with a poor response to inhaled corticosteroids $(3,4)$. In adult asthma patients, airway neutrophil counts are associated with the severity of the disease $(5,6)$. Indeed, neutrophils have been demonstrated to play a critical role in airway inflammation in patients with acute and severe asthma $(5,7,8)$. Therefore, neutrophil-predominant inflammation frequently occurs during asthma exacerbation, or in steroid-refractory patients, while the molecular mechanisms has not been determined.

Effective immune regulation depends on the balance between cell proliferation and death (9). Neutrophilic asthma is characterized by massive influx of neutrophils into the airway. Delayed apoptosis and prolonged survival of neutrophils are the potential mechanisms contributing to the persistent airway neutrophilia in severe asthma (10). Surprisingly, although glucocorticoids are usually recognized as anti-inflammatory, administration of glucocorticoids to neutrophilic asthmatic patients aggravates lung inflammation, since glucocorticoids augment the survival of neutrophils by delaying their apoptosis and ultimate clearance from lung tissues (11). Several therapies targeting neutrophils had been developed for the treatment of neutrophilic asthmatic patients, such as a CXCR2 antagonist (12), TNF-a antagonist (13-15), and a human anti-IL-17A monoclonal antibody (16). However, these therapies usually result in unsatisfactory responses or appear to cause mechanism-related side-effects, limiting their clinical application. Thus, the necessity of developing novel effective treatments for neutrophilic asthma is appealing.

Neutrophils constitute the first line defense during pulmonary infection, by efficiently binding, engulfing, and killing pathogens through phagocytosis, degranulation, and specifically release of neutrophil extracellular traps (NETs) (17). NETs are weblike structures comprising DNA and antimicrobial proteins, ejected from neutrophils undergoing a specific form of cell death called NETosis (18). Recently, extracellular DNA traps have been identified in both eosinophilic and neutrophilic asthmatic airways (19). However, subjects with neutrophilic asthma had higher neutrophil counts and NETs than eosinophil counts and eosinophil extracellular traps (EETs) (20). This difference is likely due to the fact that in neutrophilic asthma, especially in its severe form, neutrophil apoptosis is delayed, and clearance of the cell debris dysfunctioned, leading to an extensive accumulation of NETs $(10,11,21)$. High extracellular DNA concentrations in sputum mark a subset of patients with more severe asthma $(22,23)$. NETs are generally believed critical for killing bacteria,

Abbreviations: NETs, neutrophil extracellular traps; EETs, eosinophil extracellular traps; Nec-1, necrostatin-1; RIP1, receptor-interacting protein 1; PMA, phorbol 12-myristate 13-acetate; PBNs, peripheral blood neutrophils; LDH, lactate dehydrogenase; OVA, ovalbumin; CFA, complete Freund's adjuvant; BAL, bronchoalveolar lavage; RIP1, receptor-interacting protein 1; kinase; MPO, myeloperoxidase; AHR, airway hyperresponsiveness; PAF, platelet-activating factor; RIPK3/MLKL, receptor-interacting protein 3 kinase/mixed-lineage kinase domain-like protein; DAMPs, damage-associated molecular patterns. but excessive NETs may be harmful $(21,24)$. So regulation of neutrophils releasing NETs may be a therapeutic target for neutrophilic inflammation in asthma.

Necrostatin-1 (Nec-1) has been reported as an inhibitor of the receptor-interacting protein 1 (RIP1) kinase and a potent and specific inhibitor of necroptosis $(25,26)$. Prior reports showed that Nec-1 could resolve inflammation by blocking RIP1 kinase activity in several experimental disease conditions, for example acute kidney injury, cardiac contractile dysfunction and brain injury after subarachnoid hemorrhage (27-29). Surprisingly, in our experiment, we unexpectedly found that Nec-1 inhibited NETs formation induced by phorbol 12-myristate 13-acetate (PMA). Therefore, in this study, we determined to investigate the exact mechanisms underlying this inhibition and its therapeutic effect on neutrophil-dominated asthma.

\section{MATERIALS AND METHODS}

This study was approved by the Ethics Committee of the Third Affiliated Hospital of Southern Medical University Trial Registration Identifier is at clinicaltrials.gov: NCT02385331.

\section{Subjects and Sputum Collection}

Asthma was diagnosed by physicians based on the Global Initiative for Asthma (GINA) guidelines. We enrolled the asthmatics and chronic cough patients, who did not use systemic or inhaled corticosteroids for at least 4 weeks or more before sputum examination. Informed written consent forms were obtained from the participants, and the protocol was approved by the ethics committee of the Third Affiliated Hospital, Southern Medical University (2016-213). Sputum was induced using isotonic saline that contained a short-acting bronchodilator as previously study described (30). Sputum was prepared as follows: all portions with visibly greater solidity were carefully selected and placed in a pre-weighed Eppendorf tube. The total cell count was determined using a hema-cytometer. The sputum cells were collected by cytocentrifugation and 500 cells were examined after staining with Diff-Quick (American Scientific Products, Chicago, USA). Sputum samples that contained $10 \%$ squamous epithelial cells were excluded from the study. The remainder of the homogenized sputum sample was centrifuged at $1,000 \mathrm{~g}$ for $5 \mathrm{~min}$, and the supernatant was collected and stored at $-70^{\circ} \mathrm{C}$ for subsequent protein analyses. Data here is from 11 adult asthma participants and 16 adult chronic participants who provided a sample of induced sputum.

\section{Neutrophil Isolation}

Blood samples, $5 \mathrm{ml}$ from each donor, were obtained from healthy donors. Neutrophils were isolated by dextran sedimentation and centrifugation as previously described (31). The cells were cultured in RPMI1640 medium containing $10 \%$ autologous serum.

\section{Induction, Isolation, and Quantification of NETs}

The NETs were induced by PMA and isolated as previously described (32) with some modifications. Peripheral blood 
neutrophils (PBNs) were isolated from healthy donors, seeded onto six-well plates $\left(9 \times 10^{6}\right.$ cells/well $)$, and treated with $500 \mathrm{nM}$ of PMA for $3 \mathrm{~h}$. Supernatants were carefully suctioned and discarded, and neutrophils on the bottom of each dish washed by pipetting a total of $15 \mathrm{ml}$ per dish of cold PBS in order to remove all adherent cells. Cells obtained from each dish were centrifuged for $10 \mathrm{~min}$ at $450 \mathrm{~g}$ at $4^{\circ} \mathrm{C}$. Neutrophils and any remaining cells would pellet at the bottom, leaving a cell-free NET-rich supernatant. The supernatants containing cell-free DNA were collected and DNA concentrations quantified using Quant-iTTM PicoGreen dsDNA kit (Invitrogen, Paisley, UK) according to the manufacturer's instructions.

\section{Cell Culture and Cell Viability Assay}

Human bronchial epithelial cell line 16HBE was donated by the Department of Pathophysiology, College of Life Sciences, Southern Medical University. The $16 \mathrm{HBE}$ cells were cultured in RPMI1640 medium containing $10 \%$ fetal calf serum. The cells were incubated in the absence or presence of various concentrations of NETs for $24 \mathrm{~h}$ and the cell viability tested by Cell Counting Kit (CCK-8) (Dojindo, Beijing, China) following the manufacturer's instructions. The concentration of extracellular lactate dehydrogenase $(\mathrm{LDH})$ released by the neutrophils was measured using the LDH kit (Promega, USA). Cytotoxicity was calculated by the level of $\mathrm{LDH}$ released from the lysed neutrophils. The fraction of detached cells was calculated as (cell number in unwashed plates-cell number in the washed plates)/cell number in unwashed plates $) \times 100 \%$. Level of the inflammatory cytokine IL-1 $\beta$ in the supernatant was assessed using an ELISA kit (R\&D Systems, USA) according to the manufacturer's protocol.

\section{Immunofluorescence Analysis of NETs Formation in vitro}

Human blood neutrophils were pretreated with or without $\mathrm{Nec}-1$ for $30 \mathrm{~min}$ in vitro, placed on cytospin and stimulated with $25 \mathrm{nM}$ PMA or PBS for $4 \mathrm{~h}$. Subsequently, the cells were fixed with $1 \mathrm{ml}$ PBS containing 1\% paraformaldehyde. After blocking with $5 \%$ fetal bovine serum, the cytospin were stained with rabbit anti-MPO (Abcam, catalog ab208670, Cambridge, UK) followed by a secondary Cy3-conjugated goat anti-rabbit IgG antibody (Servicebio, catalog GB21303, Boston, MA, USA). The DNA was counterstained by DAPI (Thermo Fisher Scientific). The specimens were mounted and analyzed using a fluorescence microscope.

Hoechst 33342 staining and microscopy were used to confirm the presence of morphological characteristic of neutrophils. Neutrophils isolated from peripheral blood of healthy donors were seeded in six-well plates at $5 \times 10^{6}$ cells/well and cultured for $3 \mathrm{~h}$ in 1,640 medium containing with $50 \mu \mathrm{M} \mathrm{Nec}-1,25 \mathrm{nM}$ PMA, or $50 \mu \mathrm{M} \mathrm{Nec-1} \mathrm{together} \mathrm{with} 25 \mathrm{nM}$ PMA. Subsequently, the cells were stained with Sytox Green and Hoechst 33342 for $15 \mathrm{~min}$ and analyzed by fluorescence microscopy.

Neutrophils isolated from peripheral blood of health donors were seeded in six-well plates at $5 \times 10^{6}$ cells/well and the cells treated with $25 \mathrm{nM}$ PMA or $50 \mu \mathrm{M} \mathrm{Nec}-1$ together with $25 \mathrm{nM}$ PMA for $30 \mathrm{~min}$. FITC-marked non-membrane nucleic acid dye Sytox Green was used as DNA marker. Dynamic changes of neutrophil NETs after PMA stimulation were recorded in two channels, light field channel (measuring changes in cell morphology and structure under light microscope) and FITC green channel (measuring non-transmembrane nucleic acid dye Sytox Green. Imaging was performed with an automatic living cell imaging analysis system (Biotek Lionheart) in a temperaturecontrolled chamber at $36 \circ \mathrm{C}$. Images $(2,048 \times 2,048$ pixels $)$ were acquired at $0.5 \%$ maximal laser intensity with the first image at PMA stimulation for $30 \mathrm{~min}$ and then at every $5 \mathrm{~min}$ for each well for a total of $4 \mathrm{~h}$.

\section{Western Blotting}

Western blot analysis was performed using isolated human neutrophil. Neutrophils $\left(5 \times 10^{6}\right)$ were lysed with ice-cold RIPA (Radio Immunoprecipitation Assay) buffer containing a protease inhibitor cocktail for $30 \mathrm{~min}$ before centrifugation (14,000 rpm, $\left.4^{\circ} \mathrm{C}, 5 \mathrm{~min}\right)$. All manipulations were performed on ice. Protein content was determined by using bicinchoninic acid (BCA) assay. Protein samples $(30 \mu \mathrm{g}$ per lane) were resolved by SDS-PAGE and then transferred to polyvinylidene difluoride (PVDF) membranes. Blots were blocked with 5\% skimmed milk powder in TBS plus Tween before probing with antibodies to MLKL(ab194699, Abcam), pMLKL(ab187091,abcam) and $\beta$ actin(Sigma-Aldrich). The membranes were incubated overnight at $4{ }^{\circ} \mathrm{C}$ with an anti- MLKL polyclonal antibody (1:1000 dilution), anti-PMLKL polyclonal antibody (1:1000 dilution), and anti$\beta$-actin monoclonal antibody (1:5000 dilution; Sigma-Aldrich). Then they were incubated for $1 \mathrm{~h}$ at room temperature with an HRP-conjugated secondary antibody (1:5000 dilution; Thermo, Fremont, USA). The target protein was detected using an enhanced chemiluminescence solution (GE Healthcare, Little Chalfont, UK) on X-ray film.

\section{Experimental Animals}

Wild-type male C57BL/6 mice, aged 6-7 weeks, were purchased from the Animal Center of the Southern Medical University and housed in a conventional animal facility. All protocols for the mice were approved by the Ethics Committee for Animal Studies at the Third Affiliated Hospital of Southern Medical University.

\section{Neutrophilic Inflammation Model of Murine Asthma}

The neutrophil-dominated asthma model was established according to previously published protocols $(30,33)$. Mice were immunized by intraperitoneal injection of $20 \mu \mathrm{g}$ grade $\mathrm{V}$ chicken egg ovalbumin (OVA; Sigma-Aldrich) at days 0 and 7. OVA was dissolved in endotoxin-free $25 \mu \mathrm{L}$ of PBS emulsified in $75 \mu \mathrm{L}$ of complete Freund's adjuvant (CFA; Sigma-Aldrich). On days 14 and 15, the immunized mice were challenged for $40 \mathrm{~min}$ with an ultrasonic nebulized (DeVilbis, Somerset, PA, USA) aerosol containing 0.1\% OVA in saline (OVA/CFA group). Alternatively, the mice were challenged with the same aerosol in combination with a nasal drip of $30 \mu \mathrm{L}$ of $25 \mathrm{nM}$ PMA (OVA/CFA+PMA group). Mice assigned to the Nec-1 group were intraperitoneally injected Nec-1 (Sigma-Aldrich), $6 \mathrm{mg} / \mathrm{kg}$ body weight, $1 \mathrm{~h}$ after each OVA aerosol challenge. Control 
mice were intraperitoneally injected the same volume of saline. Twenty-four hours after the last OVA challenge, all mice were sacrificed and an autopsy performed. Following collection of BAL, the left lung was fixed in $4 \%$ paraformaldehyde, and the right lung was kept in tissue culture medium on ice before being homogenized for further processing.

\section{BAL Collection and Differential Cell Counts} BAL cells were collected by slowly injecting $0.5 \mathrm{~mL}$ of ice-cold PBS into the trachea using a 22-inch intravenous catheter and collecting the outflow fluids. The total cell number in BAL was counted with a Neubauer chamber. Additionally, cytospin specimen were prepared, stained with Wright-Giemsa, and a total of 400 cells counted and classified under a microscope to determine the fraction of eosinophils, neutrophils, lymphocytes, and macrophages. Results are expressed as the number of cells per milliliter of BAL.

\section{Evaluating the Formation of NETs}

To quantify the amount of PMA-induced formation of NETs in vitro, neutrophils isolated from human blood $(3 \times 105$ cells/well in $200 \mu \mathrm{L}$ of medium) were seeded into black, flatbottomed, 96-well plates and incubated for $4 \mathrm{~h}$ in a humidified incubator at $37^{\circ} \mathrm{C}$ with an atmosphere containing $5 \%$ of $\mathrm{CO}_{2}$. The medium was supplemented with phosphate-buffered saline

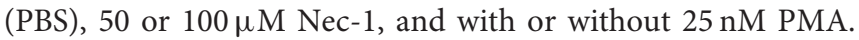
Extracellular DNA was stained with the membrane-impermeable DNA-binding dye Sytox Green (Thermo Fisher Scientific, USA). The plates were analyzed using Spectra Max M3 fluorescent plate reader (Molecular Devices) with excitation at $485 \mathrm{~nm}$ and emission at $520 \mathrm{~nm}$. The same approach was employed to measure the level of NETs in the BAL. For this purpose, $150 \mu \mathrm{L}$ of BAL per well was used.

\section{Flow Cytometric Analysis of BAL Cells}

BAL cell suspensions were obtained directly from the mice or from in vitro cultures. The expression of cell surface markers was determined by flow cytometry using fluorescent dye-conjugated mouse antibodies. Mouse neutrophils were identified by Ly6G expression. To analyze apoptosis, BAL cells were incubated with FITC-labeled annexin V and propidium iodide (MultiSciences, Hangzhou, China). Data were acquired with the FACSVerse $^{\mathrm{TM}}$ (BD Biosciences) and analyzed with the FlowJo software (version 7.6).

\section{Immunofluorescent Staining of the BAL Cells}

The BAL was collected from mouse lungs $24 \mathrm{~h}$ after the final challenge. The BAL cells were incubated on poly-L-lysine-coated coverslips for $4 \mathrm{~h}$. Subsequently, the liquid was discarded from the coverslips and the cells fixed for $15 \mathrm{~min}$ in $1 \mathrm{ml} \mathrm{PBS} / 1 \%$ paraformaldehyde. The fixed cells were then washed with icecold PBS and blocked for $90 \mathrm{~min}$ with $5 \%$ fetal bovine serum. Next, the cells were incubated with the primary antibody, anti-myeloperoxidase (MPO) or anti-cleaved caspase3 (Cleavecaspase3). Following washing, samples were incubated for $45 \mathrm{~min}$ with an Alexa 568-labeled (goat anti-rabbit) or an Alexa Fluor 488-labeled secondary antibodies (Donkey anti-goat). Coverslips were mounted onto glass slides using ProLong Gold mounting medium (Thermo Fisher Scientific, USA), sealed, and analyzed by fluorescence microscopy.

\section{Evaluation of NETs in BAL Fluid}

BAL was collected from mouse lungs $24 \mathrm{~h}$ after the final challenge and centrifuged at 1,000 rpm for $10 \mathrm{~min}$. To detect NETs, $150 \mu \mathrm{L}$ of supernatant was mixed with $1.5 \mu \mathrm{L}$ Sytox Green dye (Thermo Fisher Scientific, USA) that was diluted 1:50 in PBS.

\section{Histopathologic Evaluation of Lung Tissue}

To maintain tissue integrity, mice to be used for histologic evaluation were not subjected to bronchoalveolar lavage. Lungs were fixed in $10 \%$ formalin and embedded in paraffin. Sections were cut, stained with hematoxylin and eosin, and observed under a microscope. All slides were examined independently by two investigators blinded to the experimental treatment.

\section{Immunohistochemistry of Lung Tissue}

Lungs were removed, fixed in $4 \%$ paraformaldehyde for $4 \mathrm{~h}$, and immersed in $30 \%$ sucrose for $3-4$ days at $4^{\circ} \mathrm{C}$. Sections, $15 \mu \mathrm{m}$ thick, were cut from frozen lung tissues on a cryostat. Rabbit anticleaved caspase-3 (Asp175, diluted 1:500 in PBS; Cell Signaling Technology) and mouse anti-MPO (ab90810; diluted 1:100 in PBS; Abcam) were used as primary antibodies. The staining was visualized with a confocal laser scanning microscope (Leica DM IRE2, Germany). MPO and cleaved caspase-3 were used to identify, neutrophils and apoptotic cells, respectively. Doublepositive cells were considered to represent apoptotic neutrophils.

\section{Measurement of Airway Hyperresponsiveness}

Airway responsiveness was determined with a non-invasive method to measure mouse lung resistance after the mice were challenged with aerosolized methacholine (Sigma-Aldrich). Briefly, $24 \mathrm{~h}$ after the final OVA challenge, mice were exposed to an aerosol containing increasing concentrations of methacholine $(0,1.5625,3.125,6.25,12.5$, and $25 \mathrm{mg} / \mathrm{mL}$ in saline) using Buxco FinePointe plethysmograph (Buxco Electronics, Troy, NY). Data on lung resistance were collected continuously, and mean values represented changes in airway function.

\section{Measurement of Pro-inflammatory Cytokines in BAL}

The levels of inflammatory cytokines TNF- $\alpha$, IL- $1 \beta$, and IFN- $\gamma$ were measured with ELISA kits (R\&D Systems, USA) according to the manufacturer's protocol.

\section{Measurement of MPO}

Lung tissues were frozen in liquid nitrogen and homogenized in PBS. The homogenate was used to determine MPO according to the manufacturer's Instruction (Nanjing Jian Cheng Bioengineering Institute, China).

\section{Total Protein Concentration in BAL}

Total protein concentration in the supernatant of BAL was measured by the Bradford method with bovine serum 
albumin (Beyotime Institute of Biotechnology, China) used as the standard.

\section{Preparation of $\mathrm{Nec}-1$}

Nec-1 was purchased from Sigma-Aldrich; $5 \mathrm{mg}$ of the compound were dissolved in $0.5 \mathrm{ml} \mathrm{DMSO}$, and $100 \mu \mathrm{L}$ aliquots were stored at $-20^{\circ} \mathrm{C}$.

\section{Statistical Analysis}

Results are presented as means \pm SEM. Data were analyzed with one-tailed Student $t$-test (1-tailed) or one-way ANOVA, followed by the Tukey post-hoc test or the non-parametric Wilcoxon ranksum test. All statistical analyses were performed using the Prism7 software (GraphPad Software, La Jolla, CA, USA). $P<0.05$ was considered statistical significance.

\section{RESULTS}

\section{Sputum eDNA Levels Are Significantly Correlated With Sputum Neutrophils in Asthmatics and Chronic Cough Patients, and NETs Induced Damage of Human Airway Epithelial Cells}

It has been reported that sputum extracellular DNA (eDNA) in asthma is associated with airway neutrophilic inflammation, and increases in soluble NET components. In the study firstly we detected the levels of sputum eDNA in asthma and chronic cough patients. Sputum samples of 11 patients with asthma and 16 patients with chronic cough were collected. We found that sputum eDNA levels were significantly correlated with sputum neutrophil percentage in asthmatics and chronic cough patients (Figures 1A,B), but not significantly correlated with sputum eosinophil percentage in asthmatics and chronic cough patients (Figures 1C,D). Although excessive NETs are present in the airways of neutrophil-dominate inflammation, whether they have direct effect on bronchial epithelial cells is unclear. Therefore, different concentrations of NETs were co-cultured with human bronchial epithelial cell line 16HBEs for $24 \mathrm{~h}$. The morphology of the cells changed significantly, from a triangle or polygon to a round shape, and the cells detached from the bottom of the culture plate (Figure 1E). Cell death rate and extracellular level of LDH were significantly increased when the concentrations of NETs were more than $200 \mathrm{ng} / \mathrm{mL}$ (Figure 1F). The impact of NETs on cell viability and detachment of cells were dose-dependent (Figures 1G,H). Moreover, the inflammatory cytokine IL-1 $\beta$ in the culture supernatant was significantly increased by the treatment of 1,000 ng/mL of NETs (Figure 1I). Thus, NETs induce damage of airway epithelial cells and trigger an inflammatory response.

\section{NETs Aggravated Neutrophil-Dominated Airway Inflammation}

To determine whether NETosis and associated extracellular DNA contribute to the pathogenesis of neutrophilic asthma, the OVA/CFA induced neutrophil-dominated asthma-like airway inflammation model was employed (33), according to the schemes illustrated in Figure 2A. As expected, a substantial infiltration of neutrophils in the airways is found in the OVA/CFA induced mouse models (Figure 2C). Staining with Sytox Green revealed that the NETs in the BAL were significantly increased (Figure 2E). PMA is a strong inducer of NETs formation, therefore PMA (25 nM) was administered intranasally to induce NETs in the airways of OVA/CFA-induced mice (Figure 2B). Exposure of mice to PMA resulted in a significant increase of neutrophils and NETs in BAL compared with model mice (Figures 2D,F). Additionally, histologic examination of the lungs documented that airway inflammation was more severe in the PMA-stimulated group than OVA/CFA alone group (Figure 2G). The data suggest that NETs are involved in the pathogenesis of neutrophilic asthma and accumulation of NETs may aggravate neutrophil-dominated airway inflammation.

\section{Nec-1 Significantly Inhibited PMA-Induced NETs Formation in vitro}

Nec-1 has been recognized as a potent and specific inhibitor of necroptosis. To investigate whether Nec-1 inhibited PMAstimulated NETs formation, peripheral blood neutrophils (PBNs) isolated from healthy donors and cultured for $3 \mathrm{~h}$ in the absence (control) or presence of different concentration of Nec$1(50$ or $100 \mu \mathrm{M})$, with or without PMA $(25 \mathrm{nM})$. The results demonstrated that Nec-1 significantly inhibited PMA-induced NETs formation (Figure 3A) with representative images shown in Figure 3B. Immunofluorescent staining of MPO (a marker of neutrophils) and DAPI (staining of nuclei) further documented the inhibitory effect of Nec-1 (Figure 3C, Figure S4). The effect of Nec-1 on neutrophil releasing NETs was also examined by staining with Sytox Green and Hochest-33342. And in comparison with the group stimulated by PMA alone, less fluorescence of Sytox Green was present in cells treated with PMA (25 nM) plus Nec-1(50 $\mu \mathrm{M})$ (Figure 3D). Through these experiments confirmed that $\mathrm{Nec}-1$ inhibited PMA-stimulated NETs formation, but the exact mechanism was unclear. As Nec-1 could inhibit PMA-induced NETs formation and the amount of NETs corresponded to the damage of airway bronchial epithelial cells, we believed that Nec-1 could be used to treat neutrophildominated airway inflammation.

\section{Nec-1 Attenuated OVA/CFA-Induced Neutrophil-Dominated Airway Inflammation by Inhibiting NETs Formation}

To test whether Nec-1 can be used in the treatment of neutrophilic asthma, OVA/CFA neutrophil-dominated airway inflammation mouse model was established (shown in Figure 2A). Airway hyper-responsiveness (AHR), assessed by a non-invasive ventilator, was alleviated in Nec-1 treatment group (Figure 4A). Furthermore, we tested total cells and level of NETs in BAL. Results showed that total cells were reduced in Nec-1 treatment group (Figure 4B). Sytox Green staining revealed that the level of NETs in the BAL from OVA/CFA primed mice was reduced by Nec-1 treatment (Figure 4D). Other inflammatory indicators such as total protein concentration (an indicator of lung leakage), pulmonary MPO activity (a marker 
A

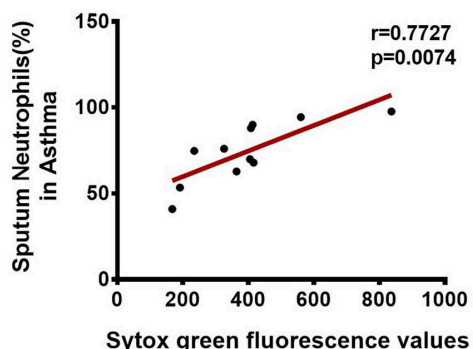

C

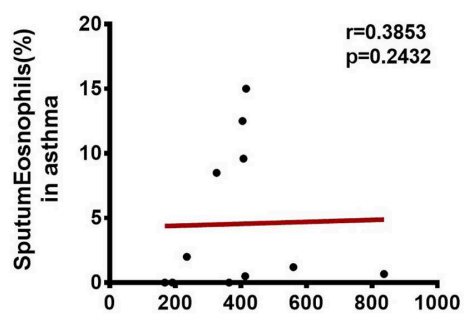

Sytox green fluorescence values

E

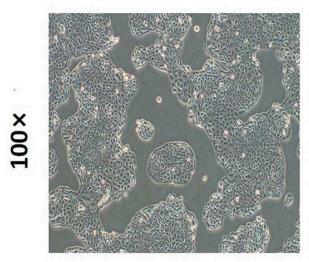

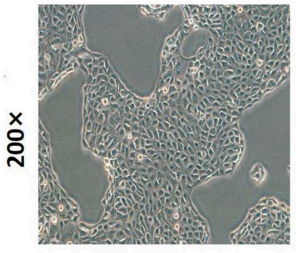

Ctrl
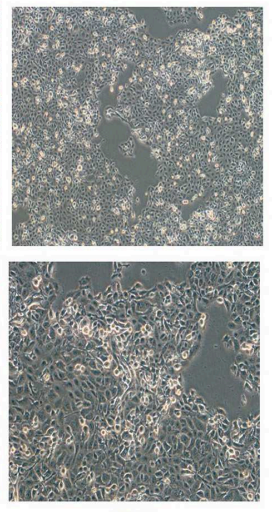

NETs

H

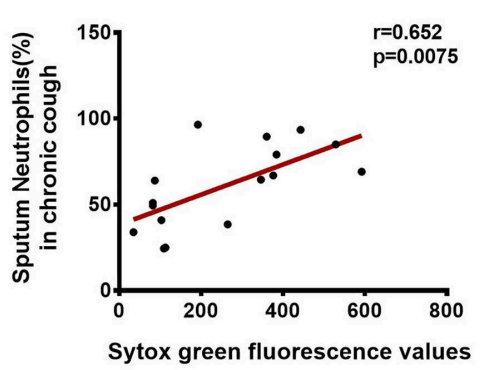

D

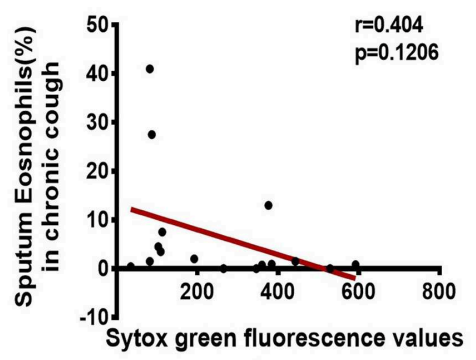

$\mathbf{F}$

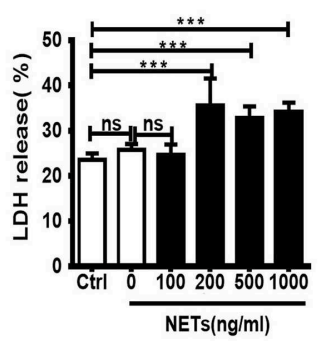

G

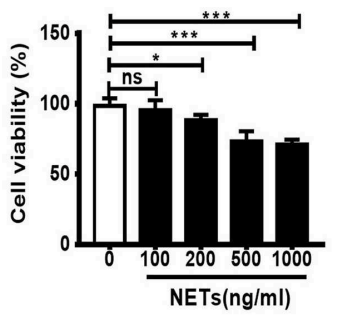

I

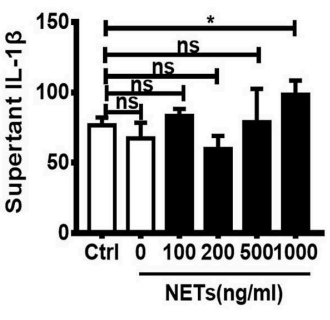

FIGURE 1 | Sputum extracellular DNA (eDNA) levels are significantly correlated with sputum neutrophils in asthmatics and chronic cough patients, and neutrophil extracellular DNA traps (NETs) induced damage of human airway epithelial cells. (A,B) Extracellular DNA in sputum stained with Sytox Green. Sputum eDNA levels were significantly correlated with sputum neutrophils percentage in asthmatics and chronic cough. (C,D) Sputum eDNA levels were not significantly correlated with sputum eosinophil percentage in asthmatics and chronic cough. (E) Morphological changes of $16 \mathrm{HBE}$ cells after treatment with $500 \mathrm{ng} / \mathrm{ml}$ of $\mathrm{NETs}$ for $24 \mathrm{~h}$. $16 \mathrm{HBEs}$ were treated with different concentrations of NETs for $24 \mathrm{~h}$, and LDH release (F), cell viability (G), detached cells (\%) (H) and supernatant interleukin (IL)-1 $\beta$ (I) were detected. The data are shown as mean \pm SD. ( ${ }^{\star} p<0.05,{ }^{\star \star \star} p<0.001$, ns, not significant). The results are representative of at least three experiments. 
A

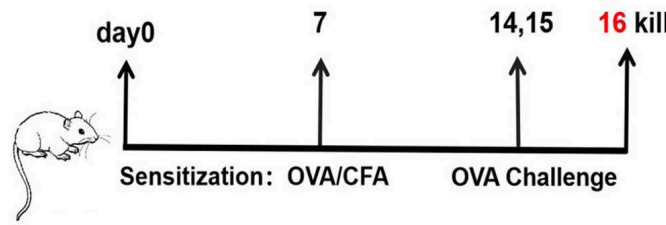

C

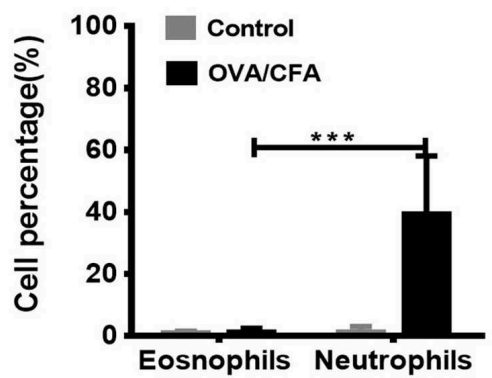

E

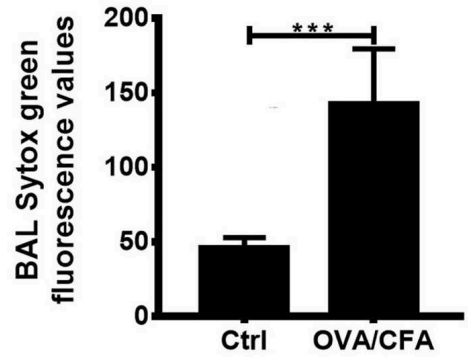

G

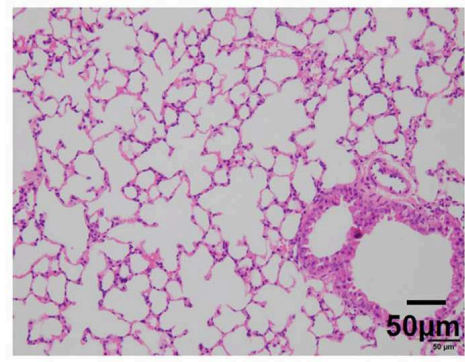

Ctrl $(200 \times)$

B

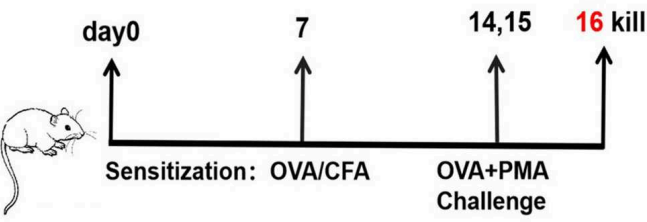

D

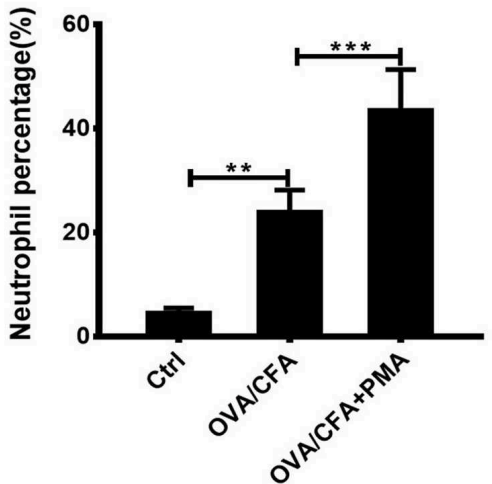

F

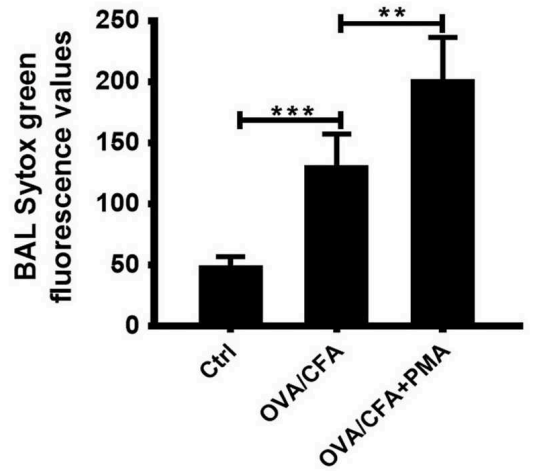

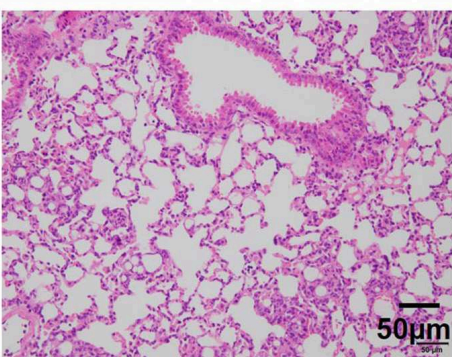

OVA/CFA $(200 \times)$

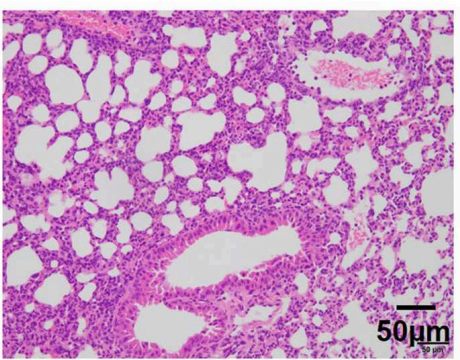

OVA/CFA+PMA $(200 \times)$

FIGURE 2 | NETs aggravated neutrophil-dominated airway inflammation. (A) Schematic diagram of OVA/CFA sensitized neutrophil-dominated airway inflammation model. (B) Schematic diagram of OVA/CFA plus PMA sensitized neutrophil-dominated airway inflammation model. (C) Pulmonary inflammatory cell populations of OVAVCFA sensitized mice with neutrophil-dominated airway inflammation. (D) The percentages of neutrophils in total BAL cells of OVA/CFA and OVA/CFA plus PMA groups. (E,F) Extracellular DNA in BAL stained with Sytox Green. (G) Representative images of H\&E-stained lung tissues of OVA/CFA and OVA/CFA plus PMA groups. Values are expressed as means \pm SEM of three independent experiments. $\left({ }^{\star \star} p<0.01,{ }^{\star \star \star} p<0.001\right)$. 
A

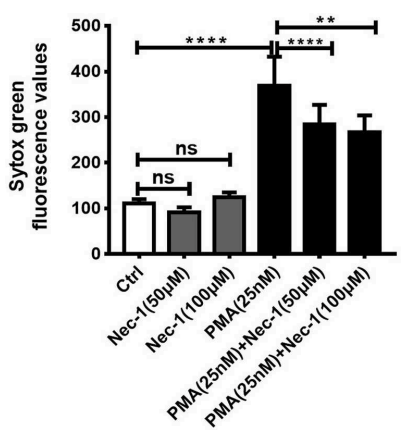

C
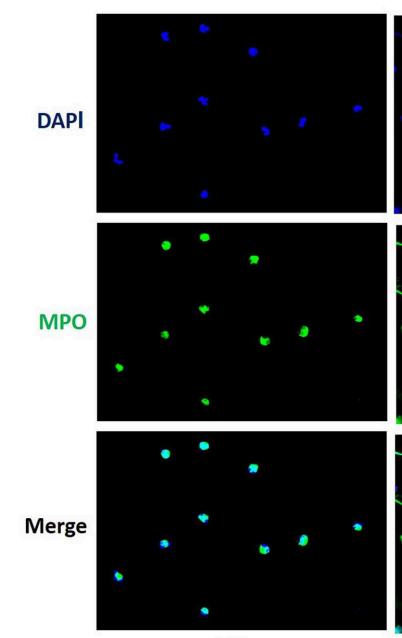

Ctrl

B

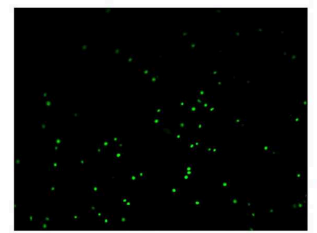

Ctrl

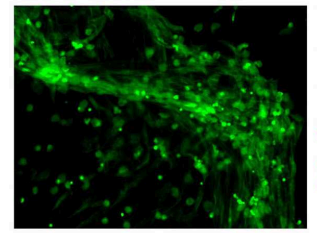

PMA
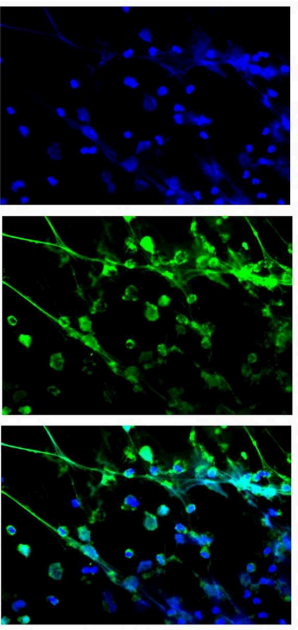

PMA

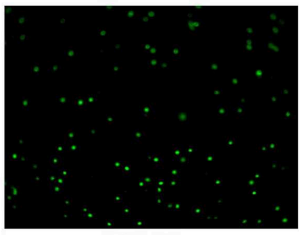

Nec-1

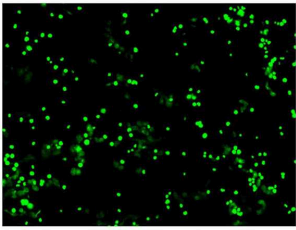

PMA+Nec-1
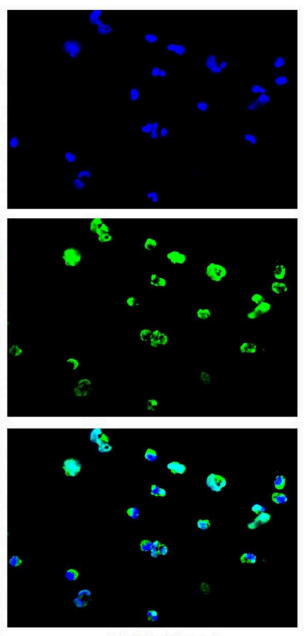

PMA+Nec-1

D

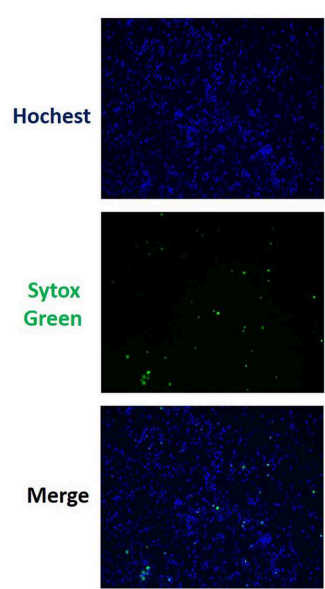

Ctrl
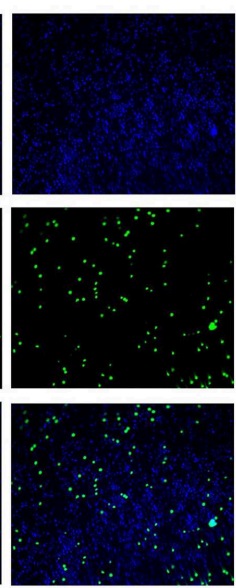

Nec-1
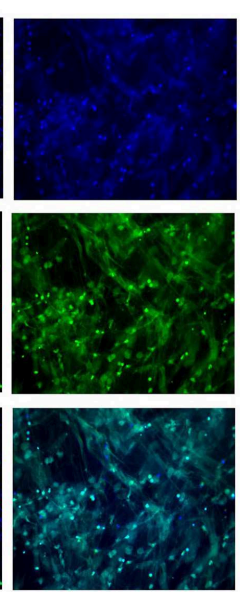

PMA
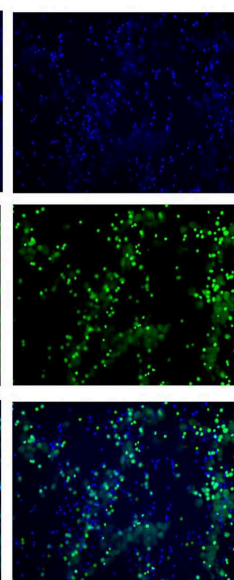

PMA+Nec-1

FIGURE 3 | Nec-1 inhibited PMA-induced NETs formation in vitro. (A) Levels of extracellular DNA released by peripheral blood neutrophils, which were cultured with PMA (25 nM) or different concentrations of Nec-1 (50 or $100 \mu \mathrm{M})$, or both. (B) Representative immunofluorescence images of Sytox Green-stained neutrophils cultured for $3 \mathrm{~h}$ in the absence (ctrl) or presence of PMA (25 nM), Nec-1 (50 $\mu \mathrm{M})$, or both $(200 \mathbf{x})$. (C) Representative immunofluorescence images of neutrophils pretreated with Nec-1 $(50 \mu \mathrm{M})$ and stimulated with or without PMA (25 nM). Upper panels illustrate DAPI (blue), central panels illustrate MPO (green), lower panels show merged images $(400 \mathbf{x})$. (D) Representative immunofluorescence images of neutrophils pretreated without (ctrl) or with Nec-1(50 $\mu$ M) and stimulated with PMA (25 nM) or both. Upper panels illustrate Hoechst-33342 (blue), central panels illustrate Sytox Green, and lower panels show merged images (200x). The values were shown as mean \pm SEM. $\left({ }^{\star *} p<0.01,{ }^{* \star *} p<0.0001\right.$, ns, not significant). The results are representative of three independent experiments. 
A

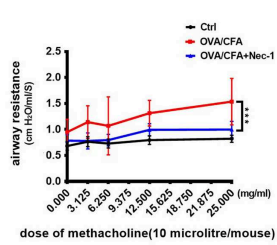

D

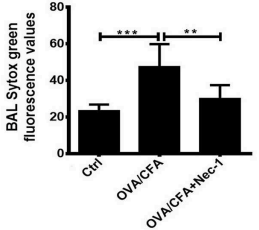

G
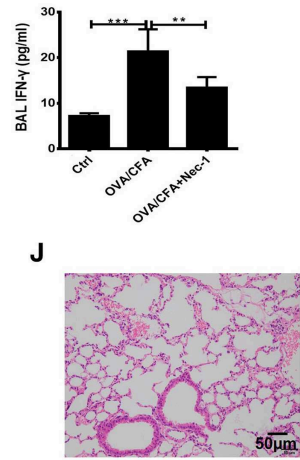

Ctrl $(200 \times$
B

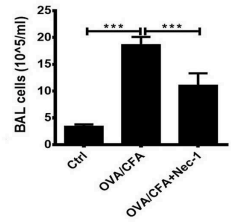

E

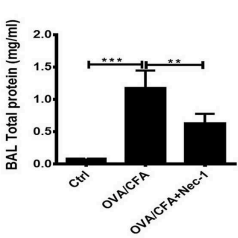

H

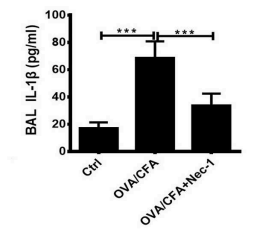

C

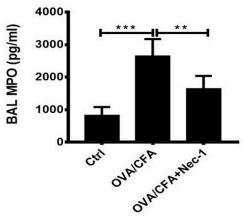

$\mathbf{F}$

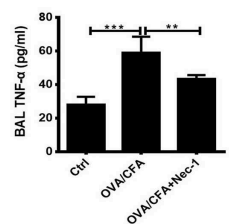

I

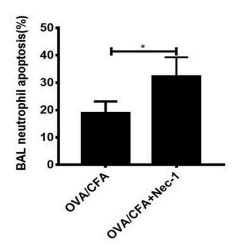

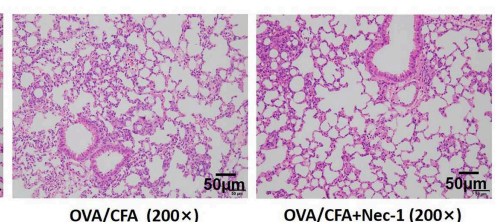

K
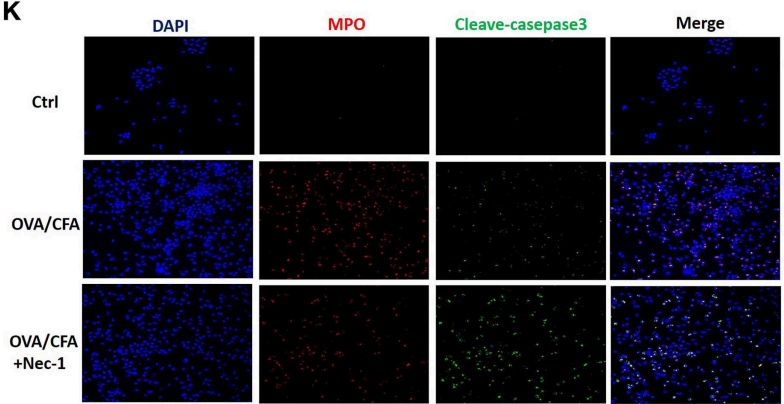

L
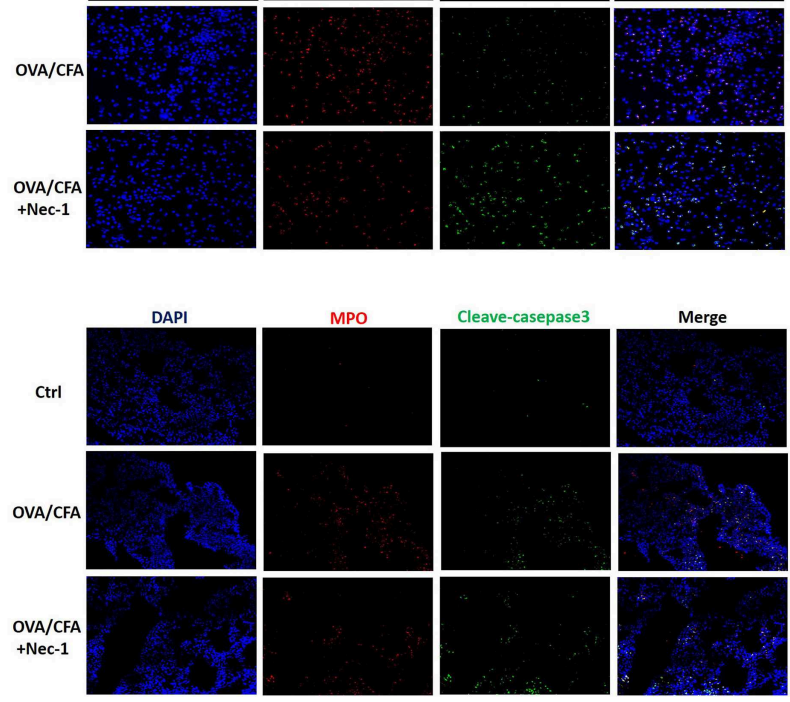

FIGURE 4 | Nec-1 attenuated OVA/CFA sensitized neutrophil-dominated airway inflammation by inhibiting NETs formation. OVA/CFA sensitized mice were treated with Nec-1 (6 mg/kg body weight), and BAL harvested. (A) OVA/CFA sensitized mice were challenged with different doses of methacholine and treated with Nec-1 (6 $\mathrm{mg} / \mathrm{kg}$ body weight), and BAL and lung tissues were harvested. Airway hyper-responsiveness used to assess the responses of mice to different doses of methacholine. (B) Total number of cells in BAL. (C) MPO activity in lung tissue homogenate. (D) Levels of extracellular DNA in BAL stained with Sytox Green. (E) Total protein concentration in BAL. (F-H) Levels of TNF- $\alpha$, IFN- $\gamma$, and IL-1 $\beta$ in BAL. (I) Fraction of apoptotic neutrophils in the BAL determined by flow cytometry. 
FIGURE 4 | Quantitative analysis of flow cytometry data ( $n \geq 3$; ${ }^{\star \star \star} P<0.001$ vs. control). (J) Representative images of H\&E-stained lung tissue of mice from different experimental groups. (J) Representative immunofluorescence images of BAL cells. From left to right, the panels illustrate staining with DAPI, MPO antibody, and cleaved caspase- 3 antibody. The right panels show the merge of images $(200 \times)$. (K,L) Representative immunofluorescence images of BAL cells and lung tissues of control, OVAVCFA, and Nec-1 groups $(200 \times)$. From left to right, the panels illustrate staining with DAPI, MPO antibody, and cleaved caspase-3 antibody. The right panels show the merge of image. Values are expressed as means \pm SEM. $\left({ }^{\star} p<0.05,{ }^{\star *} p<0.01,{ }^{\star \star *} p<0.001\right.$, ns, not significant).

of neutrophils), and pro-inflammatory mediators IL- $1 \beta$, TNF- $\alpha$, and IFN- $\gamma$ in BAL were significantly decreased in the $\mathrm{Nec}-1$ treatment group (Figures 4C,E-H). Histological examination also showed that the lung inflammation was markedly reduced by Nec-1 (Figure 4J). Thus, we speculated that Nec-1 could resolve neutrophil-dominated airway inflammation by inhibiting NETs release, but the exact mechanism underlying the inhibition of NETs formation was unclear.

Furthermore, we also found neutrophil apoptosis increased in vivo study. Neutrophil apoptosis in the BAL was analyzed by flow cytometry. Neutrophils were labeled by Ly6G antibody and apoptosis was distinguished from necrosis by FITC-labeled Annexin V and PI. In BAL of OVA/CFA primed mice, annexin $\mathrm{V}$-positive neutrophils increased significantly $(p<0.001)$ by Nec-1 treatment (Figure 4I). Additionally, immunofluorescence analysis of BAL cells demonstrated that $\mathrm{Nec}-1$ treatment group was associated with a decreased number of MPO-positive cells and an increased number of cleaved-caspase 3-positive cells (Figure 4K). Also, immunofluorescence histochemistry assay of lung tissue also showed that $\mathrm{Nec}-1$ treatment group had less MPO positive cells and more cleaved-casepase 3 positive neutrophils compared with OVA/CFA group (Figure 4L). This in vivo study further confirmed that Nec-1 reduced NETs formation and induced neutrophil apoptosis in both BAL cells and lung tissue. Thus, in vitro and in vivo studies support the notion that $\mathrm{Nec}-1$ inhibits NETosis while promoting apoptosis.

\section{Nec-1 Inhibited PMA-Induced NETs Formation by Suppressing Phosphorylation and Perforation of MLKL in vitro}

In order to explore the exact mechanism underlying the inhibition of NETs formation of Nec-1, we used an automatic living cell imaging analysis system to capture the morphological changes of the cells that were stained with cell impermeable stain Sytox Green (Videos S1-S4 respectively indicated different groups Ctrl, PMA(25nM),PMA+Nec-1(50 $\mu \mathrm{M})$ and $\mathrm{PMA}+(100 \mu \mathrm{M})$ were shown in Supplementary Files). We found that in PMA stimulation group the nuclei of neutrophils first condensed and cells rapidly depolymerized and swelled, and then the nuclei disintegrated and cell membrane collapsed. These results suggest that PMA induces degradation of DNA and pore formation in the cytoplasmic membrane, which results in membrane rupture and release of a large number of NETs. Interestingly, Nec-1 does not inhibit nuclear shrinkage, cell swelling, or chromosome depolymerization, but it dependently decreased the number of cells with membrane rupture and release of NETs (stained with Sytox Green) (Figures 5A-C). It is suggested that Nec-1 inhibits the production of NETs by protecting the integrity of cell membrane. Prior study indicated that Nec-1 selectively targets the kinase activity of RIPK1 and involved regulating RIPK3/ MLKL-dependent necroptotic death (26), so we further study the downstream molecules of RIPK1. Western-blotting experiments showed that PMA could cause MLKL phosphorylation, but Nec-1 inhibited mixed lineage kinase domain-like (MLKL) phosphorylation (Figures 5D,E, and Figure S1). As we know after phosphorylation, MLKL form a polymer to punch holes in the cell membrane and disrupt it $(34,35)$. Therefore, we believe that Nec-1 inhibits neutrophil release of NETs by suppressing phosphorylation and perforation of MLKL.

\section{DISCUSSION}

Neutrophilic asthma is characterized by a massive influx of neutrophils into the airways, which has been associated with persistent airway inflammation $(36,37)$. Although infiltration of excess neutrophils is thought to aggravate pulmonary inflammation, the detailed mechanism remains unclear. NETosis is a specific form of cell death of neutrophils that is distinct from apoptosis and other types of necrosis by releasing large web-like NETs (38). NETs are composed of DNA strands associated with histones and decorated with lots of different proteins. Although NETosis and the released NETs could provide important biological advantage for the host to fight against certain microbial infections (39). NETosis does not only occur during infection, but is also initiated by proinflammatory cytokines such as IL-8, TNF- $\alpha$, platelet-activating factor (PAF), and GM-CSF, as well as other stimulants such as LPS, PMA and complement factor 5a (C5a) (40). However, NETs act as doubleedged swords, high levels of NETs lead to more significant release of proinflammatory cytokines, further accelerating inflammation $(20,21,41)$. The presence of a large amount of NETs in the airways of neutrophilic asthma has been suggested in the pathogenesis and exacerbation of neutrophilic asthma (22-24). In our study we first demonstrated that sputum eDNA levels are significantly correlated with sputum neutrophils in asthmatics and chronic cough, but not significantly correlated with sputum eosinophils (Figures 1A-D). To determine the role of NETs in neutrophil-dominated asthma, in this study, we further assessed whether isolated NETs could damage airway epithelial cells in vitro. We found that NETs directly caused death of airway epithelial cells and resulted in the cells to release a large amount of inflammatory factors (Figures 1E-I). We also used dsDNA synthesized in vitro, and found it damage vein endothelial cells (Figure S2). Therefore, the NETosis and NETs release should be tightly regulated to avoid damage to the host.

To further study the role of NETs in asthma, a classic OVA/CFA induced allergic pulmonary inflammation model was 
A

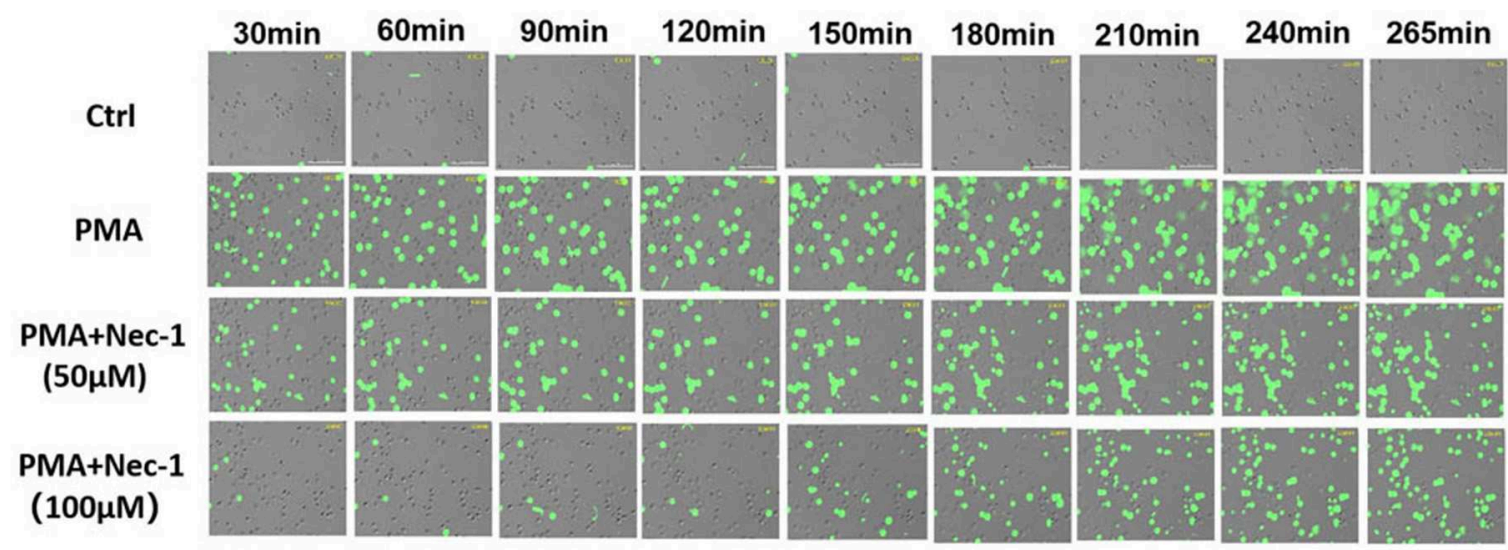

B

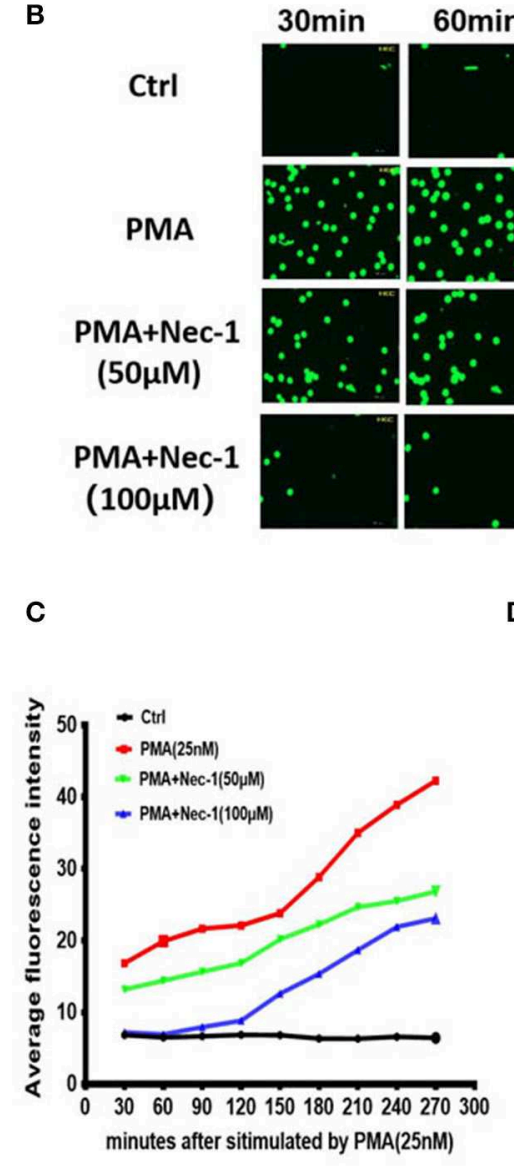

D
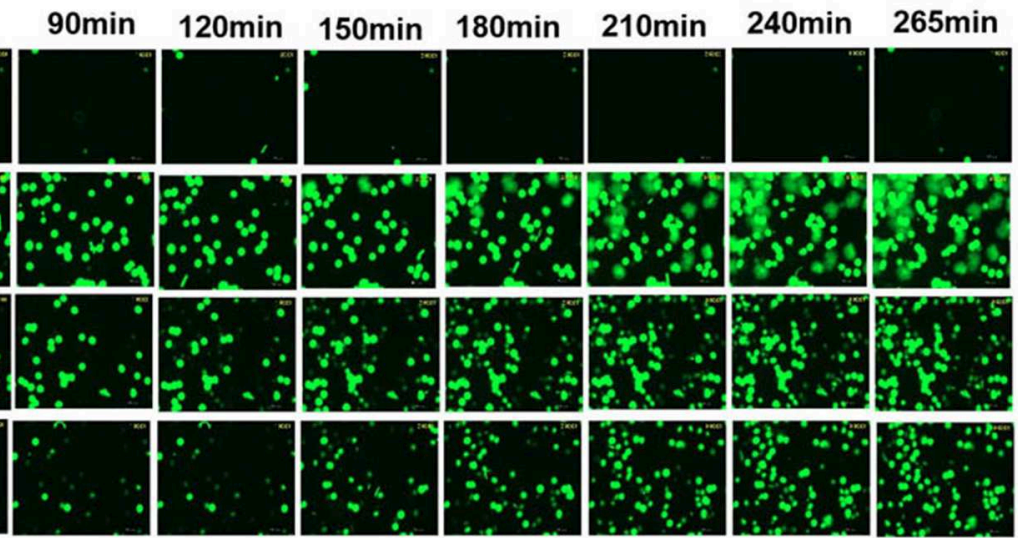

C

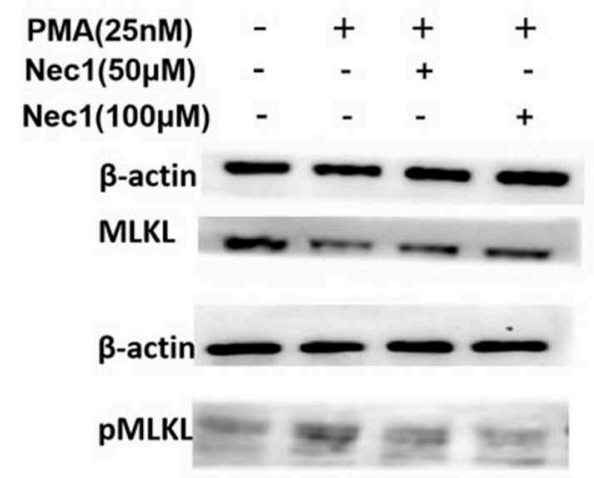

E

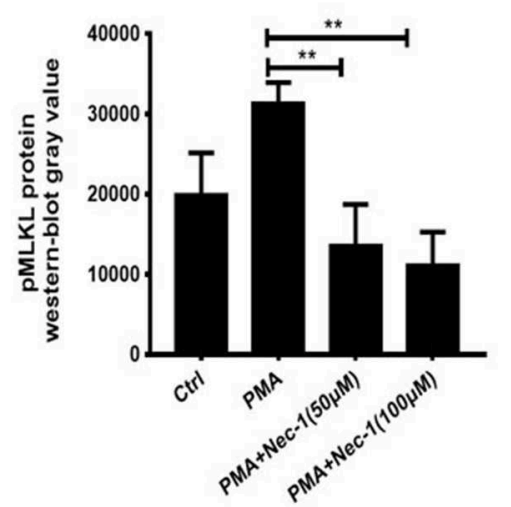

FIGURE 5 | Nec-1 inhibited PMA-induced NETs formation by suppressing phosphorylation and perforation of MLKL in vitro. (A,B) Sytox Green stained neutrophils, stimulated with $25 \mathrm{nM}$ PMA alone, or with different concentrations of Nec-1 (50 or $100 \mu \mathrm{M}$ ) and imaged for $4 \mathrm{~h}$ using an automatic live cell imaging analysis system (Videos S1-S4 respectively represent different groups Ctrl, PMA(25 nM), PMA+Nec-1(50 $\mu$ M) and PMA+(100 $\mu$ M) were shown in additional files). (A) showed light field channel (measuring changes in cell morphology and structure under ordinary optics microscope). (B) showed FITC green channel (measuring

non-transmembrane nucleic acid dye Sytox Green). The green fibrin-like structure indicated NETs. (C) The mean fluorescence intensity of Sytox Green at different time points analyzed by Image $\mathrm{J}$ semi-quantitative statistics, and the trends of NETs after PMA stimulation analyzed, as shown in Video is representative of three independent experiments. Values are expressed as means \pm SEM of three independent experiments $\left({ }^{*} p<0.05\right.$, ${ }^{* *} p<0.01$, ${ }^{* \star *} p<0.001$, ns, not significant). (D) Human neutrophils $\left(5 \times 10^{6} \mathrm{cells} / \mathrm{ml}\right)$ were treated with buffer control, PMA ( $\left.25 \mathrm{nM}\right)$ with or without different concentrations of Nec- 1 ( 50 or $\left.100 \mu \mathrm{M}\right)$ for $2 \mathrm{~h}$. After cell lysis, proteins were subjected to MLKL and pMLKL. Western Blots are representative of at least three separate experiments. (E) Westerblot results of three times with grayscale analysis and statistical analysis $\left({ }^{* *} p<0.01\right)$. 


\section{Before treatment Neutrophilic inflammation}

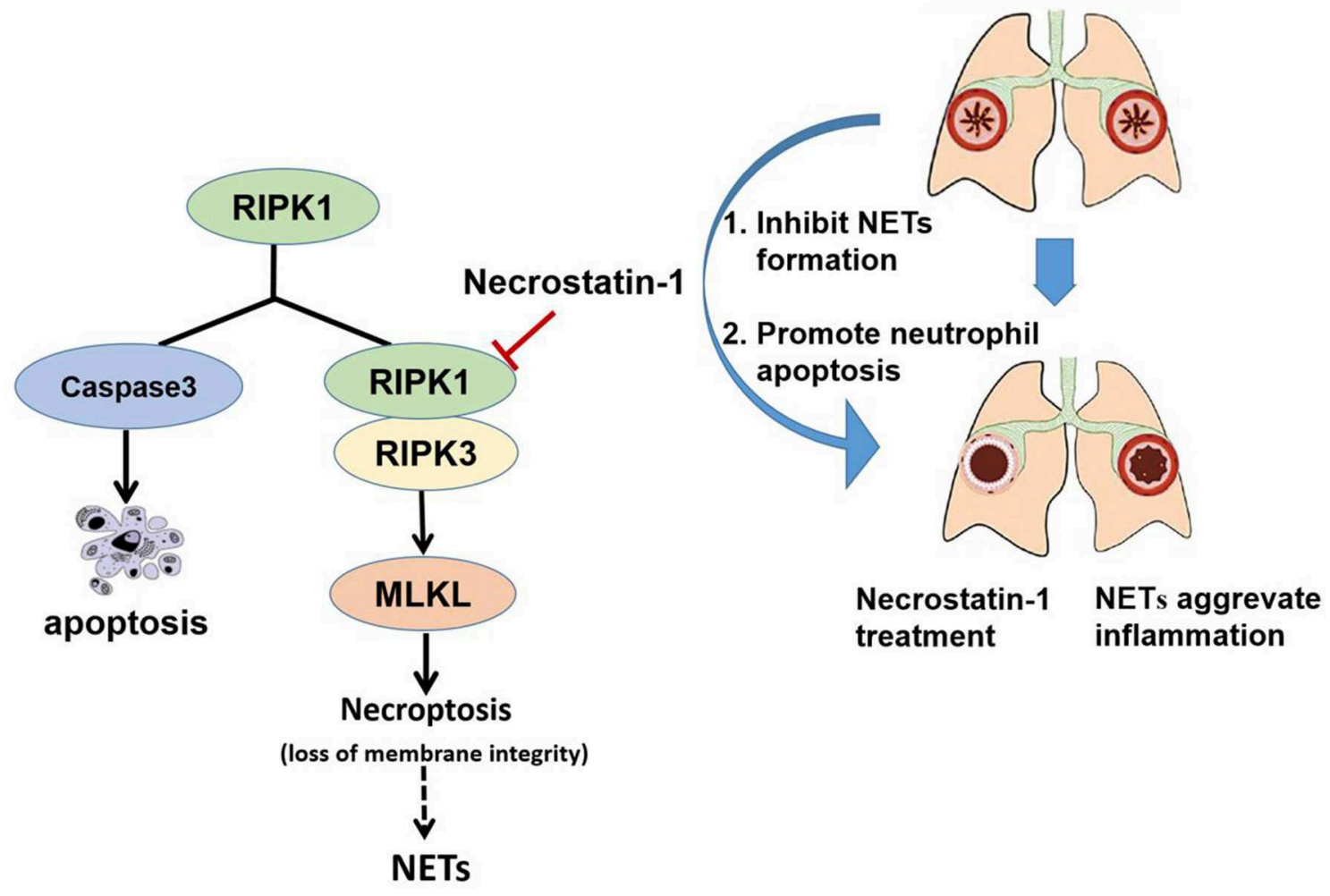

FIGURE 6 | Schematic illustration of Necrostatin-1(Nec-1) ameliorates neutrophilic inflammation by suppressing MLKL phosphorylation to inhibiting NETS release. As a necroptosis inhibitor, Nec-1 selectively targets the kinase activity of RIPK1, and thus inhibit mixed lineage kinase domain-like (MLKL) phosphorylation. We speculate that Nec-1 inhibit NETs release by inhibiting MLKL phosphorylation and perforation. Thus, on the other hand Nec-1 promote neutrophil apoptosis by increasing caspase-3 expression. The roles of Nec-1 inhibiting NETs release and promoting neutrophils apoptosis resolve neutrophilic inflammation.

selected $(23,26)$. The OVA/CFA model is mainly manifested by neutrophil infiltration, poor response to corticosteroid (42), and the participation of Th1 and Th17 cells. The mice were OVA sensitized in the presence of complete Freund's adjuvants (CFA), a potent Th-1/Th-17-skewing adjuvant commonly used in mouse models of hypersensitivity pneumonitis, and followed by OVA aerosol challenge. During current experiments, a large number of NETs were detected in the airway of OVA/CFA model mice (Figure 2E), suggesting that NETs may be closely associated with the onset of neutrophil-dominated allergic pulmonary inflammation. To test the possibility that increased NETs release leaded to the aggravation of neutrophilic asthma, PMA, a potent inducer of NETosis, was administered intranasally. High levels of NETs in the BAL was found and significantly correlated with the proportion of neutrophils in BAL and the severity of airway inflammation (Figure 2). This result demonstrates that NETs constitute one of the important factors causing progression and exacerbation of neutrophilic asthma. Both in vitro and in vivo studies have shown that NETs can cause epithelial injury in the airway and promote the occurrence and development of neutrophil-dominated asthma. Thus, accumulated data indicate that NETs can become a potential target for the treatment of neutrophil-dominated asthma. To harness the power of NETs while producing minimal damage, it is critical to maintain the right abundance of NETs formation, but reduce the accumulation of excessive NETs to avoid tissue damage.

Nec-1 was identified as a classic inhibitor of necroptosis $(26,43)$. It selectively targets the activity of receptor-interacting protein 1 kinase (RIPK1) that regulates the receptor-interacting protein 3 kinase/mixed-lineage kinase domain-like protein (RIPK3/MLKL)-dependent necroptotic death of mouse and human neutrophils. Almost all studies on Nec-1 were mainly focused on its role of the inhibition of necroptosis $(43,44)$. In our study, we found that Nec-1 inhibited PMA-induced NETs formation (shown in Figure 3, Figure S4). Our in vivo study showed that Nec-1 could alleviate airway hyperresponsiveness in OVA/CFA mouse model (Figure 4A), and also reduced the level of NETs, total protein concentration and MPO activity in BAL as well (Figures 4C-E). The levels of inflammatory factors including IL- $1 \beta$, IFN- $\gamma$, and TNF- $\alpha$ in BAL were also reduced (Figures 4F-I). Histologic examination documented the reduction of inflammation in OVA/CFA induced neutrophildominated asthma in Nec-1 treated mice (shown in Figure 4J), suggesting that administration of $\mathrm{Nec}-1$ can alleviate airway 
inflammation in neutrophil-dominated asthma by inhibiting the formation of NETs.

In order to explore the exact mechanism of $\mathrm{Nec}-1$ inhibited NETs, the automatic live cell imaging analysis system was used to further dynamically capture the morphological changes of the cells. Interestingly, we found that although $\mathrm{Nec}-$ 1 could not inhibit nuclear shrinkage, cell swelling and chromosome depolymerization caused by PMA, it could inhibit cell membrane rupture of neutrophils and protect the integrity of cell membrane (Figures 5A-C). And we further studied the downstream molecules of RIPK1. Western-blotting experiments showed that Nec-1 inhibited mixed lineage kinase domainlike (MLKL) phosphorylation (Figures 5D,E, Figure S1). Prior study indicated that the necroptotic cell death effector MLKL phosphorylation forming a polymer which translocated from the cytoplasm to the plasma membrane to perforation (45-48). Prior study indicated that MLKL phosphorylation could stimulate downstream NADPH oxidase-independent ROS production, breakdown of the nuclear membrane, and induce NETs formation (49). So we speculate that Nec-1 suppresses the release of NETs by inhibiting phosphorylation and perforation of MLKL.

In addition, we found that $\mathrm{Nec}-1$ also induced apoptosis of neutrophils in BAL and lung tissues (Figures 4K,L, Figure S3). Indeed, we demonstrated that Nec-1 increased the fraction of cleaved caspase-3 positive neutrophils in BAL and lung tissues by immunofluorescence assays (Figures $4 \mathrm{~K}, \mathrm{~L}$ ). Based on our prior studies, we have proposed a cell death recognition model" for the immune system, in which the consequences of immune responses depend on the type of cell death (50). Necrotic cells aggravate inflammation and upregulate the immune response, while apoptotic cells reduce inflammation and down-regulate immunity (51). During the process of NETosis, intracellular components are released into extracellular space, leading to the presentation of autoantigens to host immune system and the release of damage-associated molecular patterns (DAMPs) that amplify inflammation and immune responses. Thus, the "cell death recognition model" can explain the function of NETs in the pathogenesis of neutrophilic asthma. In contrast, apoptosis, a programmed cell death that maintains membrane integrity and retains toxic neutrophil contents inside the apoptotic bodies, constitutes a mechanism that limits and resolves excessive inflammatory responses. Apoptosis and efficient phagocytosis of apoptotic bodies are crucial in the resolution of inflammation. Multiple studies have demonstrated that delayed neutrophil apoptosis is inherent in neutrophil-dominated airway inflammation $(10,11)$. Thus, we believe that Nec-1 alleviates inflammation in neutrophil-dominated asthma by inhibiting NETs formation and promoting neutrophil apoptosis, resulting in down regulation of the immune response and reduction the release of inflammatory factors (Figure 6). On the contrary, recently studies indicated that glucocorticoids have no inhibitory effect on NETs formation (52), and found that glucocorticoids can enhance the potential effect of neutrophils by delaying their apoptosis (42) which may explain the poor response to glucocorticoids in patients with neutrophil-dominated asthma.

In conclusion, the current study has shown that NETs have a critical function in the occurrence and aggravation of neutrophil-dominated asthma. Additionally, the results explain the lack of effectiveness of glucocorticoid therapy in neutrophilic asthma. The inhibition of NETs formation by $\mathrm{Nec}-1$ can serve as one of the targets in the treatment of neutrophil-dominated asthma.

\section{DATA AVAILABILITY STATEMENT}

The datasets generated for this study are available on request to the corresponding author.

\section{ETHICS STATEMENT}

The studies involving human participants were reviewed and approved by The Third Affiliated Hospital, Southern Medical University. The patients/participants provided their written informed consent to participate in this study. The animal study was reviewed and approved by The Third Affiliated Hospital, Southern Medical University.

\section{AUTHOR CONTRIBUTIONS}

$\mathrm{XH}$ and $\mathrm{HJ}$ carried out most of the experiments, participated in the analysis of data, and drafted the manuscript. JHW participated in the design of the study, data analysis and interpretation, and drafted the manuscript. XZ, JZ, and JW participated in the animal experiments and performed the statistical analysis. CY, JC, and JH participated in the immunofluorescence assays and carried out the flow cytometry analysis. KL participated in the assessment of histopathological changes and revising the manuscript. ES participated in the design and coordination of the study, and finalized the manuscript. All authors read and approved the final manuscript.

\section{FUNDING}

This work was supported by grants from the National Natural Science Foundation of China (Grants No. 31600732 and 81804051), the Guangdong provincial science and technology plan (2017A030303023), Open Project of State Key Laboratory of Respiratory Disease (Grants No. SKLRD-OP-201804), and also sponsored by Tianhe district of Guangzhou city science and technology special project (Grants No. 201704KW032).

\section{ACKNOWLEDGMENTS}

The authors wish to express gratitude to their colleagues in the research group of ES and thank professor KL from Institute of Respiratory Disease Control, The first Affiliated Hospital, Guangzhou Medical College for financial support.

\section{SUPPLEMENTARY MATERIAL}

The Supplementary Material for this article can be found online at: https://www.frontiersin.org/articles/10.3389/fimmu. 2020.00666/full\#supplementary-material

Supplementary Figure 1 | $(\mathbf{A}, \mathbf{B})$ Human neutrophils $\left(5 \times 10^{6} \mathrm{cells} / \mathrm{ml}\right)$ were treated with buffer control, PMA ( $25 \mathrm{nM})$ with or without different concentrations of 
Nec-1 (50 or $100 \mu \mathrm{M}$ ) for $2 \mathrm{~h}$. After cell lysis, proteins were subjected to pMLKL. Western Blots are representative of two times of separate experiments.

Supplementary Figure 2 | Double-stranded DNA synthesized in vitro damage HUVEs. (A) Morphological changes of human umbilical vascular endothelial cells for treatment with 500 and $5,000 \mathrm{ng} / \mathrm{ml}$ DNA for $12 \mathrm{~h}$. (B) LDH release was detected after co-cultured $1 \mathrm{~h}$. (C) cell viability was detected by CCK8 for $12 \mathrm{~h}$. The data are shown as mean \pm SD. $\left({ }^{*} p<0.05,{ }^{* *} p<0.01,{ }^{* * *} p<0.001\right.$, ns, not significant). The results are representative of at least three experiments.

Supplementary Figure $\mathbf{3}$ | Nec-1 could induce neutrophils apoptosis in a dose-dependent manner. $(\mathbf{A}, \mathbf{B})$ Different concentrations of $\mathrm{Nec}-1 \mathrm{co}$-cultured with isolated human neutrophils for $18 \mathrm{~h}$. The fraction of apoptotic neutrophils detected by flow cytometry. The results are representative of three independent experiments. The values were shown as mean \pm SEM. $\left({ }^{* *} p<0.01,{ }^{* * * *} p<\right.$ 0.0001). (C) Representative pictures of flow cytometry were shown.

\section{REFERENCES}

1. Douwes J, Gibson P, Pekkanen J, Pearce N. Non-eosinophilic asthma: importance and possible mechanisms. Thorax. (2002) 57:643-8. doi: 10.1136/thorax.57.7.643

2. Shannon J, Ernst P, Yamauchi Y, Olivenstein R, Lemiere C, Foley S, et al. Differences in airway cytokine profile in severe asthma compared to moderate asthma. Chest. (2008) 133:420-26. doi: 10.1378/chest.07-1881

3. Green RH, Brightling CE, Woltmann G, Parker D, Wardlaw AJ, Pavord ID. Analysis of induced sputum in adults with asthma: identification of subgroup with isolated sputum neutrophilia and poor response to inhaled corticosteroids. Thorax. (2002) 57:875-9. doi: 10.1136/thorax.57. 10.875

4. Jatakanon A, Uasuf C, Maziak W, Lim S, Chung KF, Barnes PJ. Neutrophilic inflammation in severe persistent asthma. Am J Respir Crit Care Med. (1999) 160:1532-9. doi: 10.1164/ajrccm.160.5.9806170

5. Cheng OZ, Palaniyar N. NET balancing: a problem in inflammatory lung diseases. Front Immunol. (2013) 4:1. doi: 10.3389/fimmu.2013. 00001

6. Moore WC, Hastie AT, Li XN, Li HS, William WB, Nizar NJ, et al. Sputum neutrophil counts are associated with more severe asthma phenotypes using cluster analysis. J Allergy Clin Immunol. (2014) 133:1557-63. doi: 10.1016/j.jaci.2013.10.011

7. McDougall CM, Helms PJ. Neutrophil airway inflammation in childhood asthma. Thorax. (2006) 61:739-41. doi: 10.1136/thx.2005.057984

8. Ray A, Kolls JK. Neutrophilic inflammation in asthma and association with disease severity. Trends Immunol. (2017) 38:942-54. doi: $10.1016 /$ j.it.2017.07.003

9. Hotchkiss RS, Strasser A, McDunn JE, Swanson PE. Cell death. $N$ Engl J Med. (2009) 361:1570-83. doi: 10.1056/NEJMra09 01217

10. Uddin M, Nong G, Ward J, Seumois G, Prince LR, Wilson SJ, et al. Prosurvival activity for airway neutrophils in severe asthma. Thorax. (2010) 65:684-9. doi: 10.1136/thx.2009.120741

11. Saffar AS, Ashdown H, Gounni AS. The molecular mechanisms of glucocorticoids-mediated neutrophil survival. Curr Drug Targets. (2011) 12:556-62. doi: 10.2174/138945011794751555

12. Nair P, Gaga M, Zervas E, Alagha K, Hargreave FE, O’Byrne PM, et al. Safety and efficacy of a CXCR2 antagonist in patients with severe asthma and sputum neutrophils: a randomized, placebo-controlled clinical trial. Clin Exp Allergy. (2012) 42:1097-3. doi: 10.1111/j.1365-2222.2012.04014.x

13. Holgate ST, Noonan M, Chanez P, Busse W, Dupont L, Pavord I, et al. Efficacy and safety of etanercept in moderate-to-severe asthma: a randomised, controlled trial. Eur Respir J. (2011) 37:1352-9. doi: 10.1183/09031936.00063510

14. Wenzel SE, Barnes PJ, Bleecker ER, Bousquet J, Busse W, Dahlén SE, et al. A randomized, double-blind, placebo-controlled study of tumor necrosis factoralpha blockade in severe persistent asthma. Am J Respir Crit Care Med. (2009) 179:549-58. doi: 10.1164/rccm.200809-1512OC
Supplementary Figure 4 | HL-60 could differentiate into mature neutrophils by all trans retinoic acid (ATRA). (A) HL-60 was induced by all trans retinoic acid (ATRA) $1 \mu \mathrm{M}$ for 3 days to differentiate into mature neutrophils, and the nuclei changed from round to paging nuclei. (B) Representative immunofluorescence of HL-60 and ATRA induced HL-60 cells stimulated with PMA (25 nM). (C) Representative immunofluorescence of $\mathrm{HL}-60$ and ATRA induced $\mathrm{HL}-60$ cells stimulated with PMA (25 nM), SAU (Staphylococcus aureus), and A23187(Calcium ion carrier).

\section{Video S1 | Indicate Ctrl group.}

Video S2 | Indicate PMA(25nM) group.

Video S3 | Indicate PMA(25 nM) + Nec-1 $(50 \mu \mathrm{M})$ group.

Video S4 | Indicate PMA(25 nM) + Nec-1(100 $\mu$ M) group.

15. Erin EM, Leaker BR, Nicholson GC, Tan AJ, Green LM, Neighbour H, et al. The effects of a monoclonal antibody directed against tumor necrosis factor-alpha in asthma. Am J Respir Crit Care Med. (2006) 174:753-62. doi: 10.1164/rccm.200601-072OC

16. Busse WW, Holgate S, Kerwin E, Chon Y, Feng J, Lin J, et al. Randomized, double-blind, placebo-controlled study of brodalumab, a human anti-IL-17 receptor monoclonal antibody, in moderate to severe asthma. Am J Respir Crit Care Med. (2013) 188:1294-302. doi: 10.1164/rccm.201212-2318OC

17. Yang $\mathrm{H}$, Biermann $\mathrm{MH}$, Brauner $\mathrm{J} \mathrm{M}$, Liu $\mathrm{Y}$, Zhao $\mathrm{Y}$, Herrmann M. New insights into neutrophil extracellular traps: mechanisms of formation and role in inflammation. Front Immunol. (2016) 7:302. doi: 10.3389/fimmu.2016.00302

18. Brinkmann V, Reichard U, Goosmann C, Fauler B, Uhlemann Y, Weiss DS, et al. Neutrophil extracellular traps kill bacteria. Science. (2004) 303:1532-5. doi: $10.1126 /$ science. 1092385

19. Dworski R, Simon HU, Hoskins A, Yousefi S. Eosinophil and neutrophil extracellular DNA traps in human allergic asthmatic airways. J Allergy Clin Immunol. (2011) 127:1260-6. doi: 10.1016/j.jaci.2010.12.1103

20. Wright TK, Gibson PG, Simpson JL, McDonald VM, Wood LG, Baines KJ. Neutrophil extracellular traps are associated with inflammation in chronic airway disease. Respirology. (2016) 21:467-75. doi: 10.1111/resp.12730

21. Porto BN, Stein RT. Neutrophil extracellular traps in pulmonary diseases: too much of a good thing? Front Immunol. (2016) 7:311. doi: 10.3389/fimmu.2016.00311

22. Marrah EL, Eleanor MD, Annabelle RC, Wilfred R, Mark RL, Michael CP, et al. Extracellular DNA, neutrophil extracellular traps, and inflammasome activation in severe asthma. Am J Respir Crit Care Med. (2019) 199:1076-85. doi: $10.1164 / \mathrm{rccm} .201810-1869$ OC

23. Uddin M, Watz H, Malmgren A, Pedersen F. NETopathic inflammation in chronic obstructive pulmonary disease and severe asthma. Front Immunol. (2019) 10:47. doi: 10.3389/fimmu.2019.00047

24. Twaddell SH, Baines KJ, Grainge C, Gibson PG. The emerging role of neutrophil extracellular traps in respiratory disease. Chest. (2019) 156:774-82. doi: 10.1016/j.chest.2019.06.012

25. Degterev A, Huang Z, Boyce M, Li Y, Prakash J, Noboru M, et al. Chemical inhibitor of nonapoptotic cell death with therapeutic potential for ischemic brain injury. Nat Chem Biol. (2005) 1:112-9. doi: 10.1038/nchembio711

26. Vandenabeele P, Grootjans S, Callewaert N, Takahashi N. Necrostatin-1 blocks both RIPK1 and IDO: consequences for the study of cell death in experimental disease models. Cell Death Differ. (2013) 20:185-7. doi: 10.1038/cdd. 2012.151

27. Zhang L, Feng Q, Wang T. Necrostatin-1 protects against paraquatinduced cardiac contractile dysfunction via RIP1-RIP3-MLKL dependent necroptosis pathway. Cardiovasc Toxicol. (2018) 18:346-55. doi: 10.1007/s12012-017-9441-Z

28. Dong W, Li Z, Chen Y, Zhang L, Zhiming Y, Huaban L, et al. Necrostatin-1 attenuates sepsis-associated acute kidney injury by promoting autophagosome elimination in renal tubular epithelial cells. Mol Med Rep. (2018) 17:3194-99. doi: $10.3892 / \mathrm{mmr} .2017 .8214$ 
29. Chen F, Su X, Lin Z, Lin Y, Lianghong Y, Jiawei C, et al. Necrostatin1 attenuates early brain injury after subarachnoid hemorrhage in rats by inhibiting necroptosis. Neuropsychiatr Dis Treat. (2017) 13:1771-82. doi: 10.2147/NDT.S140801

30. Lee TH, Chang HS, Bae DJ, Song HJ, Myung SK, Jong SP, et al. Role of S100A9 in the development of neutrophilic inflammation in asthmatics and in a murine model. Clin Immunol. (2017) 183:158-66. doi: 10.1016/j.clim.2017.08.013

31. Caudrillier A, Kessenbrock K, Gilliss BM, Nguyen JX, Marisa BM, Marc M, et al. Platelets induce neutrophil extracellular traps in transfusion-related acute lung injury. J Clin Invest. (2012) 122:2661-71. doi: 10.1172/JCI61303

32. Najmeh S, Cools-Lartigue J, Giannias B, Spicer J, Lorenzo EF. Simplified human neutrophil extracellular traps (nets) isolation and handling. J Vis Exp. (2015) e52687. doi: 10.3791/52687

33. Bogaert P, Naessens T, de Koker S, Hennuy B, Jonathan H, Muriel S, et al. Inflammatory signatures for eosinophilic vs neutrophilic allergic pulmonary inflammation reveal critical regulatory checkpoints. Am J Physiol Lung Cell Mol Physiol. (2011) 300:679-90. doi: 10.1152/ajplung.00202.2010

34. Hildebrand JM, Tanzer MC, Lucet IS, Young SN, Sukhdeep KS, Pooja S, et al. Activation of the pseudokinase MLKL unleashes the four-helix bundle domain to induce membrane localization and necroptotic cell death. Proc Natl Acad Sci USA. (2014) 111:15072-7. doi: 10.1073/pnas.1408987111

35. Liu S, Liu H, Johnston A, Hanna-Addams S, Eduardo R, Yougui X, et al. MLKL forms disulfide bond-dependent amyloid-like polymers to induce necroptosis. Proc Natl Acad Sci USA. (2017) 114:E7450-9. doi: 10.1073/pnas.1707531114

36. Choi AS, Jang JS, Park SW, Park SH, Sang HP, Jai SP, et al. Role of neutrophils in persistent airway obstruction due to refractory asthma. Respirology. (2012) 17:322-9. doi: 10.1111/j.1440-1843.2011.02097.x

37. Jang AS, Kwon HS, Cho YS, Bae YJ, Tae BK, Jong SP, et al. Identification of sub-types of refractory asthma in Korean patients by cluster analysis. Lung. (2013) 191:87-93. doi: 10.1007/s00408-012-9430-8

38. Takei H, Araki A, Watanabe H, Ichinose A, Sendo F. Rapid killing of human neutrophils by the potent activator phorbol 12-myristate 13-acetate (PMA) accompanied by changes different from typical apoptosis or necrosis. J Leukoc Biol. (1996) 59:229-40. doi: 10.1002/jlb.59.2.229

39. Sinuhe H, Stavros G, Chanchal SC. Modulation of neutrophil NETosis: interplay between infectious agents and underlying host physiology. Semin Immunopathol. (2013) 35:439-53. doi: 10.1007/s00281-013-0380-x

40. Paul H, Stavros G, Sinuhe H. Neutrophil extracellular traps in health and disease. Swiss Med Wkly. (2016) 146:w14352. doi: 10.4414/smw.2016.14352

41. Giuseppe AR, Angelo AM, Patrizia RQ, Norma M. Bet on NETs! or on how to translate basic science into clinical practice. Front Immunol. (2016) 7:417. doi: 10.3389/fimmu.2016.00417

42. Tian BP, Xia LX, Bao ZQ, Hao Z, ZhiWX, Yuan YM, et al. Bcl-2 inhibitors reduce steroid-insensitive airway inflammation. J Allergy Clin Immunol. (2017) 140:418-30. doi: 10.1016/j.jaci.2016.11.027
43. Smith CC, Yellon DM. Necroptosis, necrostatins and tissue injury. J Cell Mol Med. (2011) 15:1797-806. doi: 10.1111/j.1582-4934.2011.01341.x

44. Hitomi J, Christofferson DE, Ng A, Yao J, Alexei D, Ramnik JX, et al. Identification of a molecular signaling network that regulates a cellular necrotic cell death pathway. Cell. (2008) 135:1311-23. doi: $10.1016 /$ j.cell.2008.10.044

45. Murphy JM, Czabotar PE, Hildebrand JM, Lucet IS, Jian GZ, Silvia $\mathrm{AD}$, et al. The pseudokinase mlkl mediates necroptosis via a molecular switch mechanism. Immunity. (2013) 39:443-53. doi: 10.1016/j.immuni.2013. 06.018

46. Yoon S, Kovalenko A, Bogdanov K, Wallach D. MLKL, the protein that mediates necroptosis, also regulates endosomal trafficking and extracellular vesicle generation. Immunity. (2017) 47:51-65.e7. doi: 10.1016/j.immuni.2017.06.001

47. Petrie EJ, Sandow JJ, Jacobsen AV, Smith BJ, Michael DWG, Isabelle SL, et al. Conformational switching of the pseudokinase domain promotes human MLKL tetramerization and cell death by necroptosis. Nat Commun. (2018) 9:2422. doi: 10.1038/s41467-01804714-7

48. McNamara DE, Quarato G, Guy CS, Green DR, Moldoveanu T. Characterization of MLKL-mediated plasma membrane rupture in necroptosis. J Vis Exp. (2018) 58088. doi: 10.3791/58088

49. D'Cruz AA, Speir MB, Bliss-Moreau M, Sylvia D, Shu W, Alyce $\mathrm{AC}$, et al. The pseudokinase MLKL activates PAD4-dependent NET formation in necroptotic neutrophils. Sci Signal. (2018) eaao1716. doi: 10.1126/scisignal.aao1716

50. Sun, E. Cell death recognition model for the immune system. Med Hypotheses. (2008) 70:585-96. doi: 10.1016/j.mehy.2007.05.049

51. Sun EW, Shi YF. Apoptosis: the quiet death silences the immune system. Pharmacol Ther. (2001) 92:135-45. doi: 10.1016/S0163-7258(01)00164-4

52. Lande R, Ganguly D, Facchinetti V, Frasca L, Curdin C, Josh G, et al. Neutrophils activate plasmacytoid dendritic cells by releasing self-dna peptide complexes in systemic lupus erythematosus. Sci Transl Med. (2011) 3:73ra19. doi: $10.1126 /$ scitranslmed.3001180

Conflict of Interest: The authors declare that the research was conducted in the absence of any commercial or financial relationships that could be construed as a potential conflict of interest.

Copyright (C) 2020 Han, Jie, Wang, Zhang, Wang, Yu, Zhang, He, Chen, Lai and Sun. This is an open-access article distributed under the terms of the Creative Commons Attribution License (CC BY). The use, distribution or reproduction in other forums is permitted, provided the original author(s) and the copyright owner(s) are credited and that the original publication in this journal is cited, in accordance with accepted academic practice. No use, distribution or reproduction is permitted which does not comply with these terms. 